\title{
Probing CPT in transitions with entangled neutral kaons
}

\section{J. Bernabeu, ${ }^{a}$ A. Di Domenico ${ }^{b}$ and P. Villanueva-Perez ${ }^{a, c}$}

${ }^{a}$ Department of Theoretical Physics, University of Valencia, and IFIC, Univ. Valencia-CSIC, E-46100 Burjassot, Valencia, Spain

${ }^{b}$ Department of Physics, Sapienza University of Rome, and INFN Sezione di Roma, P.le A. Moro, 2, I-00185 Rome, Italy

${ }^{c}$ Paul Scherrer Institut, Villigen, Switzerland

E-mail: jose.bernabeu@uv.es, antonio.didomenico@roma1.infn.it, pablo.villanueva@uv.es

ABSTRACT: In this paper we present a novel CPT symmetry test in the neutral kaon system based, for the first time, on the direct comparison of the probabilities of a transition and its CPT reverse. The required interchange of in $\leftrightarrow$ out states for a given process is obtained exploiting the Einstein-Podolsky-Rosen correlations of neutral kaon pairs produced at a $\phi$ factory. The observable quantities have been constructed by selecting the two semileptonic decays for flavour tag, the $\pi \pi$ and $3 \pi^{0}$ decays for CP tag and the time orderings of the decay pairs. The interpretation in terms of the standard Weisskopf-Wigner approach to this system, directly connects CPT violation in these observables to the violating $\Re \delta$ parameter in the mass matrix of $\mathrm{K}^{0}-\overline{\mathrm{K}}^{0}$, a genuine CPT violating effect independent of $\Delta \Gamma$ and not requiring the decay as an essential ingredient.

Possible spurious effects induced by $\mathrm{CP}$ violation in the decay and/or a violation of the $\Delta S=\Delta Q$ rule have been shown to be well under control. The proposed test is thus fully robust, and might shed light on possible new CPT violating mechanisms, or further improve the precision of the present experimental limits. It could be implemented at the DA $\Phi$ NE facility in Frascati, where the KLOE-2 experiment might reach a statistical sensitivity of $\mathcal{O}\left(10^{-3}\right)$ on the newly proposed observable quantities.

Keywords: Discrete and Finite Symmetries, Kaon Physics, CP violation

ArXiv EPrint: 1509.02000 


\section{Contents}

1 Introduction 1

2 Definition of states 2

3 CPT symmetry test at a $\phi$-factory 4

4 Impact of the approximations on the test. Results. 10

$\begin{array}{lll}5 & \text { Conclusions } & 15\end{array}$

\section{Introduction}

CPT symmetry, i.e. the symmetry under the combination of charge conjugation $(\mathrm{C})$, parity $(\mathrm{P})$, and time reversal $(\mathrm{T})$ transformations, at present appears to be the only discrete symmetry of Quantum Mechanics respected in Nature experimentally. This result has a very solid theoretical foundation in the well known CPT theorem [1-4] (see also [5-7] for some more recent developments), ensuring exact CPT invariance for any quantum field theory formulated on flat space-time assuming (1) Lorentz invariance, (2) Locality, and (3) Hermiticity.

A violation of the CPT symmetry would have a dramatic impact on our present theoretical picture and would definitely constitute an unambiguous signal of a New Physics framework, thus strongly motivating both experimental searches and theoretical studies on this subject.

CPT invariance has been confirmed by all present experimental tests (see Tests of conservations laws and CPT invariance tests in neutral kaon decay reviews in [8]), particularly in the neutral kaon system where strong limits have been set to a variety of possible CPT violation effects which might arose in a quantum gravity scenario [9-13]. The best limits on the $\delta$ parameter expressing CPT violation in the $\mathrm{K}^{0}-\overline{\mathrm{K}}^{0}$ mixing matrix, i.e. in the standard Weisskopf-Wigner approach [14] (see also e.g. appendix A of [15], appendix I of [16], or [17]) are obtained in the CPLEAR experiment for $\Re \delta$ [18], and using the BellSteinberger relation for $\Im \delta[19,20]$, yielding a stringent limit on the difference of mass terms for $\mathrm{K}^{0}$ and $\overline{\mathrm{K}}^{0}:\left|m_{\mathrm{K}^{0}}-m_{\overline{\mathrm{K}}^{0}}\right|<4 \times 10^{-19} \mathrm{GeV}$ at $95 \%$ c.l. [8].

The CPT violating probe has been, however, often limited to a difference of masses (and other intrinsic properties) for a particle and its anti-particle, i.e. to diagonal mass terms. In many physical phenomena the perturbing effect does not appear at first order in perturbation theory: it would be sufficient that the perturbation breaks a symmetry of the non-perturbed states. This vanishing effect at first order for the diagonal elements, like e.g. the case of the electric dipole moment for $\mathrm{T}$ violation, is not present for transitions (non-diagonal elements) [21]. 
In this paper we discuss a new kind of CPT test for transitions in the neutral kaon system where the exchange of in and out states (and CP conjugation), required for a direct and genuine CPT test, is performed exploiting the entanglement of the kaon pair produced at a $\phi$-factory. This methodology has been recently proposed for a direct test of the $\mathrm{T}$ symmetry in the same context [22], similarly to the one adopted for the performed test in the $\mathrm{B}$ meson system at B-factories [21, 23-26]. The decay is not an essential ingredient for a non-vanishing effect and it is only used for filtering the appropriate initial and final states of the neutral kaon transition [27]. Explicitly, in the standard Weisskopf-Wigner approach to this system, our CPT-violating effects can be connected to the $\Re \delta$ parameter, a genuine CPT-violating effect independent of $\Delta \Gamma=\Gamma_{S}-\Gamma_{L}$, with $\Gamma_{S}$ and $\Gamma_{L}$ the widths of the physical states (see section 2).

Using this method it would be possible for the first time to directly test the CPT symmetry in transition processes between meson states, rather than comparing masses, lifetimes, or other intrinsic properties of particle and anti-particle states. Any CPT test requires a very clear theoretical formulation and very clean experimental conditions in order to avoid possible fake effects mimicking a CPT violation signal, e.g. possible and uncontrolled direct $\mathrm{CP}$ violation contributions that could alter the precise identification of the filtered meson state. One has to be aware that a significant result in a CPT test will involve small numbers, so that a precise control of the experimental conditions is mandatory. The test proposed in this paper exploits the peculiarities of the neutral kaon system and would allow a very clean and direct test of the CPT symmetry. This is in contrast with the problems encountered in the neutral B meson system, where one has very severe limitations to a possible clean test of CPT due to the use of the decay B $\rightarrow J / \Psi \mathrm{K}_{\mathrm{L}}$, with the current identification of $\mathrm{K}_{\mathrm{L}}$ which is not an exact $\mathrm{CP}$ eigenstate.

The KLOE-2 experiment [28] at DA $\Phi$ NE, the Frascati $\phi$-factory, might perform for the first time this kind of test reaching a sensitivity of $\mathcal{O}\left(10^{-3}\right)$ in the experimental asymmetry, a high sensitivity which - we emphasize - is a direct test by comparing a given transition with its CPT-transformed transition, and not a result of fitting a parameter in a given model. If interpreted in terms of the sensitivity to the usual CPT-violating $\delta$ parameter for the $\mathrm{K}^{0}-\overline{\mathrm{K}}^{0}$ system, it would also allow to further improve the present limits on $\delta$.

In section 2 we precisely define the in and out kaon states involved in the time evolution of the system. In section 3 we construct the CPT-violating observables using appropriate ratios of transition probabilities. In section 4 we discuss the impact of the approximations and the orthogonality problem for the states filtered by the decay channel, and evaluate the sensitivity of the proposed test. Finally in section 5 we present our conclusions.

\section{Definition of states}

In order to formulate a possible CPT symmetry test with neutral kaons, it is necessary to precisely define the different states involved as possible in and out states in the time evolution. First, let us consider the short- and long-lived physical states $\left|\mathrm{K}_{\mathrm{S}}\right\rangle$ and $\left|\mathrm{K}_{\mathrm{L}}\right\rangle$, i.e. the states with definite masses $m_{S, L}$ and lifetimes $\tau_{S, L}$ which evolve as a function of 
the kaon proper time $t$ as pure exponentials

$$
\begin{aligned}
\left|\mathrm{K}_{\mathrm{S}}(t)\right\rangle & =e^{-i \lambda_{S} t}\left|\mathrm{~K}_{\mathrm{S}}\right\rangle \\
\left|\mathrm{K}_{\mathrm{L}}(t)\right\rangle & =e^{-i \lambda_{L} t}\left|\mathrm{~K}_{\mathrm{L}}\right\rangle .
\end{aligned}
$$

with $\lambda_{S, L}=m_{S, L}-i \Gamma_{S, L} / 2$, and $\Gamma_{S, L}=\left(\tau_{S, L}\right)^{-1}$. They are usually expressed in terms of the flavor eigenstates $\left|\mathrm{K}^{0}\right\rangle,\left|\overline{\mathrm{K}}^{0}\right\rangle$ as:

$$
\begin{aligned}
& \left|\mathrm{K}_{\mathrm{S}}\right\rangle=\frac{1}{\sqrt{2\left(1+\left|\epsilon_{S}\right|^{2}\right)}}\left[\left(1+\epsilon_{S}\right)\left|\mathrm{K}^{0}\right\rangle+\left(1-\epsilon_{S}\right)\left|\overline{\mathrm{K}}^{0}\right\rangle\right] \\
& \left|\mathrm{K}_{\mathrm{L}}\right\rangle=\frac{1}{\sqrt{2\left(1+\left|\epsilon_{L}\right|^{2}\right)}}\left[\left(1+\epsilon_{L}\right)\left|\mathrm{K}^{0}\right\rangle-\left(1-\epsilon_{L}\right)\left|\overline{\mathrm{K}}^{0}\right\rangle\right],
\end{aligned}
$$

with $\epsilon_{S}$ and $\epsilon_{L}$ two small complex parameters describing the CP impurity in the physical states. One can equivalently define $\epsilon \equiv\left(\epsilon_{S}+\epsilon_{L}\right) / 2$, and $\delta \equiv\left(\epsilon_{S}-\epsilon_{L}\right) / 2$; adopting a suitable phase convention (e.g. the $\mathrm{Wu}$-Yang phase convention [29]) $\epsilon \neq 0$ implies T violation, $\delta \neq 0$ implies CPT violation, while $\delta \neq 0$ or $\epsilon \neq 0$ implies $\mathrm{CP}$ violation.

Let us also consider the states $\left|\mathrm{K}_{+}\right\rangle,\left|\mathrm{K}_{-}\right\rangle$defined as follows: $\left|\mathrm{K}_{+}\right\rangle$is the state filtered by the decay into $\pi \pi\left(\pi^{+} \pi^{+}\right.$or $\left.\pi^{0} \pi^{0}\right)$, a pure $\mathrm{CP}=+1$ state; analogously $\left|\mathrm{K}_{-}\right\rangle$is the state filtered by the decay into $3 \pi^{0}$, a pure $\mathrm{CP}=-1$ state. Their orthogonal states correspond to the states which cannot decay into $\pi \pi$ or $3 \pi^{0}$, defined, respectively, as

$$
\begin{aligned}
\left|\widetilde{\mathrm{K}}_{-}\right\rangle & \equiv \widetilde{\mathrm{N}}_{-}\left[\left|\mathrm{K}_{\mathrm{L}}\right\rangle-\eta_{\pi \pi}\left|\mathrm{K}_{\mathrm{S}}\right\rangle\right] \\
\left|\widetilde{\mathrm{K}}_{+}\right\rangle & \equiv \widetilde{\mathrm{N}}_{+}\left[\left|\mathrm{K}_{\mathrm{S}}\right\rangle-\eta_{3 \pi^{0}}\left|\mathrm{~K}_{\mathrm{L}}\right\rangle\right]
\end{aligned}
$$

with

$$
\begin{aligned}
\eta_{\pi \pi} & =\frac{\left\langle\pi \pi|T| \mathrm{K}_{\mathrm{L}}\right\rangle}{\left\langle\pi \pi|T| \mathrm{K}_{\mathrm{S}}\right\rangle} \\
\eta_{3 \pi^{0}} & =\frac{\left\langle 3 \pi^{0}|T| \mathrm{K}_{\mathrm{S}}\right\rangle}{\left\langle 3 \pi^{0}|T| \mathrm{K}_{\mathrm{L}}\right\rangle},
\end{aligned}
$$

and $\widetilde{\mathrm{N}}_{ \pm}$two suitable normalization factors. With these definitions of states, $\left|\mathrm{K}_{+}\right\rangle$and $\left|\mathrm{K}_{-}\right\rangle$ can be explicitly constructed imposing the conditions $\left\langle\widetilde{\mathrm{K}}_{ \pm} \mid \mathrm{K}_{\mp}\right\rangle=0$ :

$$
\begin{aligned}
& \left|\mathrm{K}_{+}\right\rangle=\mathrm{N}_{+}\left[\left|\mathrm{K}_{\mathrm{S}}\right\rangle+\alpha\left|\mathrm{K}_{\mathrm{L}}\right\rangle\right] \\
& \left|\mathrm{K}_{-}\right\rangle=\mathrm{N}_{-}\left[\left|\mathrm{K}_{\mathrm{L}}\right\rangle+\beta\left|\mathrm{K}_{\mathrm{S}}\right\rangle\right]
\end{aligned}
$$

where

$$
\begin{aligned}
& \alpha=\frac{\eta_{\pi \pi}^{\star}-\left\langle\mathrm{K}_{\mathrm{L}} \mid \mathrm{K}_{\mathrm{S}}\right\rangle}{1-\eta_{\pi \pi}^{\star}\left\langle\mathrm{K}_{\mathrm{S}} \mid \mathrm{K}_{\mathrm{L}}\right\rangle}, \\
& \beta=\frac{\eta_{3 \pi^{0}}^{\star}-\left\langle\mathrm{K}_{\mathrm{S}} \mid \mathrm{K}_{\mathrm{L}}\right\rangle}{1-\eta_{3 \pi^{0}}^{\star}\left\langle\mathrm{K}_{\mathrm{L}} \mid \mathrm{K}_{\mathrm{S}}\right\rangle},
\end{aligned}
$$

and $\mathrm{N}_{ \pm}$are two normalization factors.

Here we have kept separate definitions of the filtered states $\left|K_{+}\right\rangle$and $\left|K_{-}\right\rangle$, which are observed through their decay, from the tagged states $\left|\widetilde{\mathrm{K}}_{+}\right\rangle$and $\left|\widetilde{\mathrm{K}}_{-}\right\rangle$, which are prepared 
exploiting the entanglement of the kaon pairs, as we will discuss in the next section. $\left|\mathrm{K}_{+}\right\rangle$ and $\left|\mathrm{K}_{-}\right\rangle$are defined as the filtered states when observing definite $\mathrm{CP}= \pm 1$ decay products. Even though the decay products are orthogonal, the filtered $\left|\mathrm{K}_{+}\right\rangle$and $\left|\mathrm{K}_{-}\right\rangle$states can still be nonorthoghonal. In the following we will assume

$$
\begin{aligned}
\left|\mathrm{K}_{+}\right\rangle & \equiv\left|\widetilde{\mathrm{K}}_{+}\right\rangle \\
\left|\mathrm{K}_{-}\right\rangle & \equiv\left|\widetilde{\mathrm{K}}_{-}\right\rangle,
\end{aligned}
$$

which corresponds to impose the condition of orthogonality $\left\langle\mathrm{K}_{-} \mid \mathrm{K}_{+}\right\rangle=0$, implying that $\beta=-\eta_{\pi \pi}$ and $\alpha=-\eta_{3 \pi^{0}}$, and a precise relationship between the two amplitude ratios $\eta_{\pi \pi}$ and $\eta_{3 \pi^{0}}$ :

$$
\begin{aligned}
\eta_{\pi \pi}+\eta_{3 \pi^{0}}^{\star}-\eta_{\pi \pi} \eta_{3 \pi^{0}}^{\star}\left\langle\mathrm{K}_{\mathrm{L}} \mid \mathrm{K}_{\mathrm{S}}\right\rangle & =\left\langle\mathrm{K}_{\mathrm{S}} \mid \mathrm{K}_{\mathrm{L}}\right\rangle \\
& =\frac{\epsilon_{L}+\epsilon_{S}^{\star}}{\sqrt{\left(1+\left|\epsilon_{L}\right|^{2}\right)\left(1+\left|\epsilon_{S}\right|^{2}\right)}},
\end{aligned}
$$

Neglecting terms of $\mathcal{O}\left(\epsilon^{3}\right)$ (with $\epsilon=\mathcal{O}\left(10^{-3}\right)$ ), therefore with a high degree of accuracy, $\mathcal{O}\left(10^{-9}\right)$, this translates into the following relation:

$$
\eta_{\pi \pi}+\eta_{3 \pi^{0}}^{\star}=\epsilon_{L}+\epsilon_{S}^{\star}
$$

This clearly indicates that direct $\mathrm{CP}$ and $\mathrm{CPT}$ violation have to be neglected when imposing assumption (2.12). In fact, for instance, eq. (2.14) cannot be simultaneously satisfied for $\pi^{+} \pi^{-}$and $\pi^{0} \pi^{0}$ decays, being $\left(\eta_{\pi^{+} \pi^{-}}-\eta_{\pi^{0} \pi^{0}}\right)=3 \epsilon^{\prime}$, with $\epsilon^{\prime}=\mathcal{O}\left(10^{-6}\right)$ the direct CP violation parameter [8]. Similar subtle points were previously discussed in the literature for the T-asymmetry measurement in the flavour-CP eigenstates of $J / \Psi \mathrm{K}^{0}$ decay channels of $\mathrm{B}_{\mathrm{d}}$ 's [30], as well as for any pair of decay channels [31].

More in general, while possible direct CPT violation contributions might be still cast into the definition of the observable quantities for the CPT test that will be presented in the next section, direct $\mathrm{CP}$ violation may appear as a contaminating fake effect which is necessary to keep well under control.

Finally the validity of the $\Delta S=\Delta Q$ rule will be assumed in the following, so that the two flavor orthogonal eigenstates $\left|\mathrm{K}^{0}\right\rangle$ and $\left|\overline{\mathrm{K}}^{0}\right\rangle$ are identified by the charge of the lepton in semileptonic decays. When the decay into $\pi^{-} \ell^{+} \nu$ is observed, it cannot come from $\left|\overline{\mathrm{K}}^{0}\right\rangle$ so that the state $\left|\mathrm{K}^{0}\right\rangle$ is filtered, and vice-versa for the decay into $\pi^{+} \ell^{-} \bar{\nu}$.

The relevance of these assumptions will be discussed in section 4, where it will be shown that they can be safely released for our purposes, without affecting the cleanliness of the test.

\section{CPT symmetry test at a $\phi$-factory}

Similarly to the T symmetry test proposed at a $\phi$-factory (or B-factory) [22-25], the implementation of the CPT test proposed here exploits the Einstein-Podolsky-Rosen (EPR) [32] entanglement of the neutral meson pair produced in $\phi \rightarrow \mathrm{K}^{0} \overline{\mathrm{K}}^{0}$ decays. In fact in this case 


\begin{tabular}{|c|c|c|c|}
\hline Reference & T-conjug. & CP-conjug. & CPT-conjug. \\
\hline $\mathrm{K}^{0} \rightarrow \mathrm{K}_{+}$ & $\mathrm{K}_{+} \rightarrow \mathrm{K}^{0}$ & $\overline{\mathrm{K}}^{0} \rightarrow \mathrm{K}_{+}$ & $\mathrm{K}_{+} \rightarrow \overline{\mathrm{K}}^{0}$ \\
$\mathrm{~K}^{0} \rightarrow \mathrm{K}_{-}$ & $\mathrm{K}_{-} \rightarrow \mathrm{K}^{0}$ & $\overline{\mathrm{K}}^{0} \rightarrow \mathrm{K}_{-}$ & $\mathrm{K}_{-} \rightarrow \overline{\mathrm{K}}^{0}$ \\
$\overline{\mathrm{K}}^{0} \rightarrow \mathrm{K}_{+}$ & $\mathrm{K}_{+} \rightarrow \overline{\mathrm{K}}^{0}$ & $\mathrm{~K}^{0} \rightarrow \mathrm{K}_{+}$ & $\mathrm{K}_{+} \rightarrow \mathrm{K}^{0}$ \\
$\overline{\mathrm{K}}^{0} \rightarrow \mathrm{K}_{-}$ & $\mathrm{K}_{-} \rightarrow \overline{\mathrm{K}}^{0}$ & $\mathrm{~K}^{0} \rightarrow \mathrm{K}_{-}$ & $\mathrm{K}_{-} \rightarrow \mathrm{K}^{0}$ \\
\hline
\end{tabular}

Table 1. Scheme of possible reference transitions and their associated T, CP or CPT conjugated processes accessible at a $\phi$-factory.

the initial state of the pair is totally antisymmetric ${ }^{1}$ and can be written in terms of any pair of orthogonal states, e.g. $\mathrm{K}^{0}$ and $\overline{\mathrm{K}}^{0}$, or $\mathrm{K}_{+}$and $\mathrm{K}_{-}$, as:

$$
|i\rangle=\frac{1}{\sqrt{2}}\left\{\left|\mathrm{~K}^{0}\right\rangle\left|\overline{\mathrm{K}}^{0}\right\rangle-\left|\overline{\mathrm{K}}^{0}\right\rangle\left|\mathrm{K}^{0}\right\rangle\right\}=\frac{1}{\sqrt{2}}\left\{\left|\mathrm{~K}_{+}\right\rangle\left|\mathrm{K}_{-}\right\rangle-\left|\mathrm{K}_{-}\right\rangle\left|\mathrm{K}_{+}\right\rangle\right\}
$$

Thus, exploiting the perfect anticorrelation of the state implied by eq. (3.1), which remains unaltered - even in the presence of $\mathrm{K}^{0}-\overline{\mathrm{K}}^{0}$ mixing - until one of the two kaons decays, it is possible to have a "flavor-tag" or a "CP-tag", i.e. to prepare a definite $\mathrm{K}^{0}$ or $\overline{\mathrm{K}}^{0}$ state, or a definite $\mathrm{K}_{+}$or $\mathrm{K}_{-}$state of the still alive kaon by observing a specific flavor decay ${ }^{2}$ $\left(\ell^{-}\right.$or $\left.\ell^{+}\right)$or CP decay $\left(3 \pi^{0}\right.$ or $\left.\pi \pi\right)$ of the other (and first decaying) kaon in the pair. For instance, the transition $\mathrm{K}^{0} \rightarrow \mathrm{K}_{+}$and its associated probability $P\left[\mathrm{~K}^{0}(0) \rightarrow \mathrm{K}_{+}(\Delta t)\right]$ corresponds to the observation of a $\ell^{-}$decay at a proper time $t_{1}$ of the opposite $\overline{\mathrm{K}}^{0}$ and a $\pi \pi$ decay at a later proper time $t_{2}=t_{1}+\Delta t$, with $\Delta t>0$.

In other words, the $\ell^{-}$decay of a kaon on one side prepares (tags), in the quantum mechanical sense, the opposite (if undecayed) kaon in the state $\left|K^{0}\right\rangle$ at a starting time $t=0$. The $\left|\mathrm{K}^{0}\right\rangle$ state freely evolves in time until its $\pi \pi$ decay filters it in the state $\left|\mathrm{K}_{+}\right\rangle$at a time $t=\Delta t$.

In this way one can experimentally access all the four reference transitions listed in table 1, and their T, CP and CPT conjugated transitions. It can be easily checked that the three conjugated transitions correspond to different categories of events; therefore the comparisons between reference vs conjugated transitions correspond to independent $\mathrm{T}$, $\mathrm{CP}$ and CPT experimental tests. None of these transformed transitions for the three symmetries are simply an exchange of the time ordering of the two decay channels.

\footnotetext{
${ }^{1}$ It is worth noting that a possible CPT violation effect in the entangled state, the so-called $\omega$-effect [33, 34], might induce a breakdown of the antisymmetry of the initial state. The possible impact of this effect, which is strongly bounded experimentally [9-11], is not considered in this paper, for simplicity.

${ }^{2}$ In the following the semileptonic decays $\pi^{+} \ell^{-} \nu$ and $\pi^{-} \ell^{+} \bar{\nu}$ will be denoted for brevity as $\ell^{-}$and $\ell^{+}$, respectively.
} 
For the CPT symmetry test one can define the following ratios of probabilities:

$$
\begin{aligned}
R_{1, \mathrm{CPT}}(\Delta t) & =\frac{P\left[\mathrm{~K}_{+}(0) \rightarrow \overline{\mathrm{K}}^{0}(\Delta t)\right]}{P\left[\mathrm{~K}^{0}(0) \rightarrow \mathrm{K}_{+}(\Delta t)\right]} \\
R_{2, \mathrm{CPT}}(\Delta t) & =\frac{P\left[\mathrm{~K}^{0}(0) \rightarrow \mathrm{K}_{-}(\Delta t)\right]}{P\left[\mathrm{~K}_{-}(0) \rightarrow \overline{\mathrm{K}}^{0}(\Delta t)\right]} \\
R_{3, \mathrm{CPT}}(\Delta t) & =\frac{P\left[\mathrm{~K}_{+}(0) \rightarrow \mathrm{K}^{0}(\Delta t)\right]}{P\left[\overline{\mathrm{K}}^{0}(0) \rightarrow \mathrm{K}_{+}(\Delta t)\right]} \\
R_{4, \mathrm{CPT}}(\Delta t) & =\frac{P\left[\overline{\mathrm{K}}^{0}(0) \rightarrow \mathrm{K}_{-}(\Delta t)\right]}{P\left[\mathrm{~K}_{-}(0) \rightarrow \mathrm{K}^{0}(\Delta t)\right]} .
\end{aligned}
$$

The measurement of any deviation from the prediction $R_{i, \mathrm{CPT}}(\Delta t)=1$ imposed by CPT invariance is a signal of CPT violation.

It is worth noting that for $\Delta t=0$ :

$$
R_{1, \mathrm{CPT}}(0)=R_{2, \mathrm{CPT}}(0)=R_{3, \mathrm{CPT}}(0)=R_{4, \mathrm{CPT}}(0)=1,
$$

i.e. the CPT-violating effect is built in the time evolution of the system, and it is absent at $\Delta t=0$, within our approximations. Possible deviations from limits (3.3) will be discussed in the next section.

For $\Delta t \gg \tau_{S}$, assuming the presence of CPT violation only in the mass matrix $(\delta \neq 0)$ and nothing else, one gets:

$$
\begin{aligned}
& R_{2, \mathrm{CPT}}\left(\Delta t \gg \tau_{S}\right) \simeq 1-4 \Re \delta \\
& R_{4, \mathrm{CPT}}\left(\Delta t \gg \tau_{S}\right) \simeq 1+4 \Re \delta,
\end{aligned}
$$

i.e. the CPT-violating effect built in the time evolution reaches a "plateau" regime and dominates in this limit. It is a genuine effect because $\Re \delta$ does not depend on $\Delta \Gamma$ as an essential ingredient $[21,27,35]$. This constitutes an important difference with respect to the $\mathrm{T}$ symmetry test case [22], where the effect due to the $\Re \epsilon$ parameter vanishes for $\Delta \Gamma \rightarrow 0$.

At a $\phi$-factory the observable quantity is the double differential decay rate $I\left(f_{1}, t_{1} ; f_{2}, t_{2}\right)$ of the state $|i\rangle$ into decay products $f_{1}$ and $f_{2}$ at proper times $t_{1}$ and $t_{2}$, respectively [36].

After integration on $t_{1}$ at fixed time difference $\Delta t=t_{2}-t_{1} \geq 0$, the resulting decay intensity $I\left(f_{1}, f_{2} ; \Delta t\right)$ can be rewritten in a suitable form, putting in evidence the probabilities we are aiming for. In fact, for two generic pairs of orthogonal states $\left\{\mathrm{K}_{\mathrm{X}}, \overline{\mathrm{K}}_{\mathrm{X}}\right\}$ and $\left\{\mathrm{K}_{\mathrm{Y}}, \overline{\mathrm{K}}_{\mathrm{Y}}\right\}$ uniquely identified by the decay products $\left\{f_{X}, f_{\bar{X}}\right\}$ and $\left\{f_{Y}, f_{\bar{Y}}\right\}$ (e.g. the $\left\{\mathrm{K}^{0}, \overline{\mathrm{K}}^{0}\right\}$ and $\left\{\mathrm{K}_{+}, \mathrm{K}_{-}\right\}$pairs identified by the decay products $\left\{\ell^{+}, \ell^{-}\right\}$and $\left\{\pi \pi, 3 \pi^{0}\right\}$, respectively, under the assumption of eqs. (2.12) and the $\Delta S=\Delta Q$ rule), the decay intensity can be expressed as:

$$
\begin{aligned}
I\left(f_{\bar{X}}, f_{Y} ; \Delta t\right) & =\int_{0}^{\infty} I\left(f_{\bar{X}}, t_{1} ; f_{Y} ; t_{2}\right) d t_{1} \\
& =C\left(f_{\bar{X}}, f_{Y}\right) \times P\left[\mathrm{~K}_{\mathrm{X}}(0) \rightarrow \mathrm{K}_{Y}(\Delta t)\right],
\end{aligned}
$$


where the coefficient $C\left(f_{\bar{X}}, f_{Y}\right)$, depending only on the final states $f_{\bar{X}}$ and $f_{Y}$, is given by:

$$
C\left(f_{\bar{X}}, f_{Y}\right)=\frac{1}{2\left(\Gamma_{S}+\Gamma_{L}\right)}\left|\left\langle f_{\bar{X}}|T| \overline{\mathrm{K}}_{\mathrm{X}}\right\rangle\left\langle f_{Y}|T| \mathrm{K}_{Y}\right\rangle\right|^{2}
$$

and $P\left[\mathrm{~K}_{\mathrm{X}}(0) \rightarrow \mathrm{K}_{\mathrm{Y}}(\Delta t)\right]$ is the generic $\mathrm{K}_{\mathrm{X}} \rightarrow \mathrm{K}_{\mathrm{Y}}$ transition probability which contains the $\Delta t$ time dependence only.

It's worth noting that a similar expression can be easily formulated also for the case $\Delta t<0$ :

$$
I\left(f_{\bar{X}}, f_{Y} ; \Delta t\right)=C\left(f_{\bar{X}}, f_{Y}\right) \times P\left[\overline{\mathrm{K}}_{Y}(0) \rightarrow \overline{\mathrm{K}}_{\mathrm{X}}(|\Delta t|)\right] .
$$

Therefore, at a $\phi$-factory one can define the observable ratios:

$$
\begin{aligned}
R_{2, \mathrm{CPT}}^{\exp }(\Delta t) & \equiv \frac{I\left(\ell^{-}, 3 \pi^{0} ; \Delta t\right)}{I\left(\pi \pi, \ell^{-} ; \Delta t\right)} \\
R_{4, \mathrm{CPT}}^{\exp }(\Delta t) & \equiv \frac{I\left(\ell^{+}, 3 \pi^{0} ; \Delta t\right)}{I\left(\pi \pi, \ell^{+} ; \Delta t\right)},
\end{aligned}
$$

which are related to the $R_{i, \mathrm{CPT}}(\Delta t)$ ratios defined in eqs. (3.2) as follows, for $\Delta t \geq 0$ :

$$
\begin{aligned}
& R_{2, \mathrm{CPT}}^{\exp }(\Delta t)=R_{2, \mathrm{CPT}}(\Delta t) \times D_{\mathrm{CPT}} \\
& R_{4, \mathrm{CPT}}^{\exp }(\Delta t)=R_{4, \mathrm{CPT}}(\Delta t) \times D_{\mathrm{CPT}}
\end{aligned}
$$

whereas for $\Delta t<0$ one has:

$$
\begin{aligned}
& R_{2, \mathrm{CPT}}^{\exp }(\Delta t)=R_{1, \mathrm{CPT}}(|\Delta t|) \times D_{\mathrm{CPT}} \\
& R_{4, \mathrm{CPT}}^{\exp }(\Delta t)=R_{3, \mathrm{CPT}}(|\Delta t|) \times D_{\mathrm{CPT}}
\end{aligned}
$$

with $D_{\mathrm{CPT}}$ the ratio of coefficients:

$$
D_{\mathrm{CPT}}=\frac{C\left(\ell^{-}, 3 \pi^{0} ; \Delta t\right)}{C\left(\pi \pi, \ell^{-} ; \Delta t\right)}=\frac{C\left(\ell^{+}, 3 \pi^{0} ; \Delta t\right)}{C\left(\pi \pi, \ell^{+} ; \Delta t\right)}=\frac{\left|\left\langle 3 \pi^{0}|T| \mathrm{K}_{-}\right\rangle\right|^{2}}{\left|\left\langle\pi \pi|T| \mathrm{K}_{+}\right\rangle\right|^{2}}
$$

that can be expressed, with a high degree of accuracy, at least $\mathcal{O}\left(10^{-7}\right)$, as:

$$
D_{\mathrm{CPT}}=\frac{\mathrm{BR}\left(\mathrm{K}_{\mathrm{L}} \rightarrow 3 \pi^{0}\right)}{\mathrm{BR}\left(\mathrm{K}_{\mathrm{S}} \rightarrow \pi \pi\right)} \frac{\Gamma_{L}}{\Gamma_{S}} .
$$

The value of $D_{\mathrm{CPT}}$ can be therefore evaluated from branching ratios and lifetimes, but it is also directly measurable from the observable ratios (3.9) and (3.10), as it will be discussed in detail in the next section.

The explicit expressions of ratios (3.9) and (3.10) (neglecting higher order terms in small parameters and for not too large negative $\Delta t$ ) are:

$$
\begin{aligned}
R_{2, \mathrm{CPT}}^{\exp }(\Delta t) & =\frac{P\left[\mathrm{~K}^{0}(0) \rightarrow \mathrm{K}_{-}(\Delta t)\right]}{P\left[\mathrm{~K}_{-}(0) \rightarrow \overline{\mathrm{K}}^{0}(\Delta t)\right]} \times D_{\mathrm{CPT}} \\
& \simeq|1-2 \delta|^{2}\left|1+2 \delta e^{-i\left(\lambda_{S}-\lambda_{L}\right) \Delta t}\right|^{2} \times D_{\mathrm{CPT}}
\end{aligned}
$$



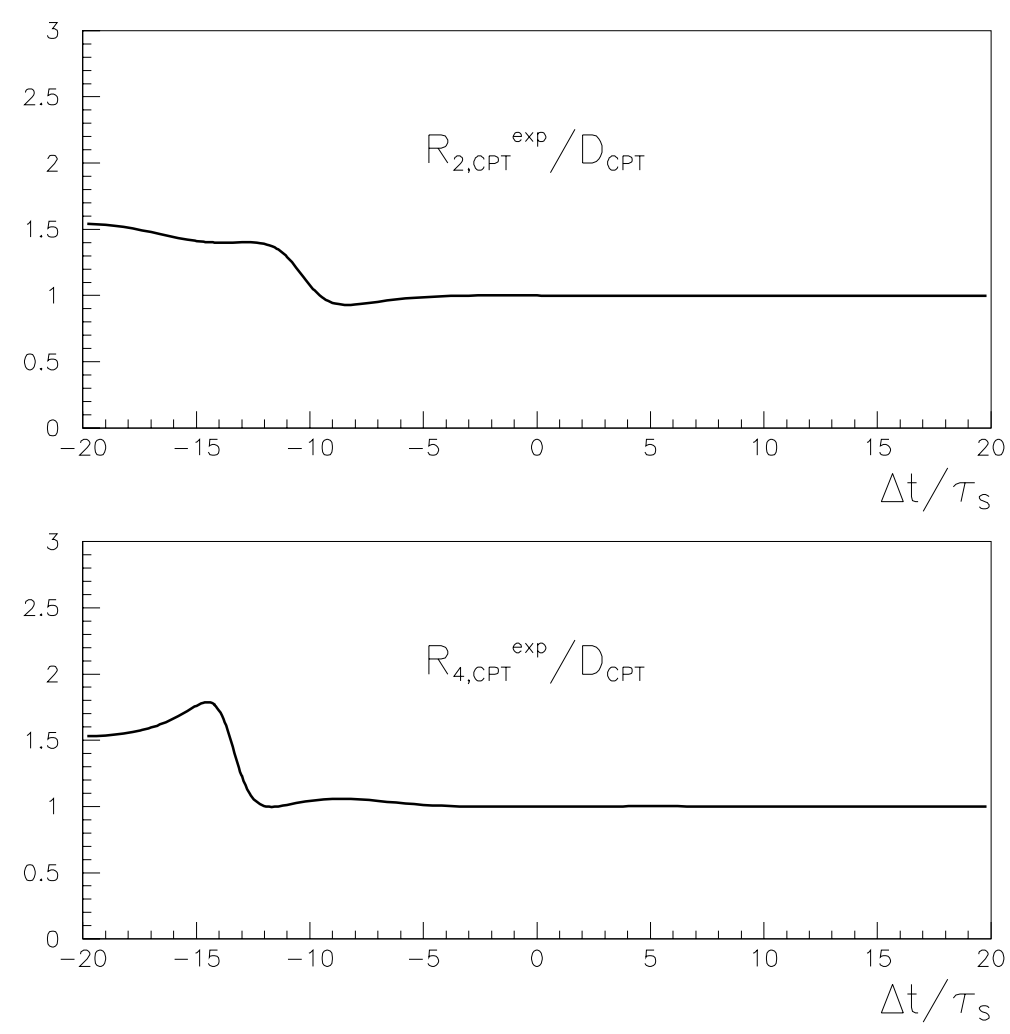

Figure 1. The ratios $R_{2, \mathrm{CPT}}^{\exp }(\Delta t)$ and $R_{4, \mathrm{CPT}}^{\exp }(\Delta t)$ as a function of $\Delta t$. For visualization purposes the CPT violating parameters have been fixed to the values $\Re \delta=3.3 \times 10^{-4}$ and $\Im \delta=1.6 \times 10^{-5}$.

$$
\begin{aligned}
R_{4, \mathrm{CPT}}^{\exp }(\Delta t) & =\frac{P\left[\overline{\mathrm{K}}^{0}(0) \rightarrow \mathrm{K}_{-}(\Delta t)\right]}{P\left[\mathrm{~K}_{-}(0) \rightarrow \mathrm{K}^{0}(\Delta t)\right]} \times D_{\mathrm{CPT}} \\
& \simeq|1+2 \delta|^{2}\left|1-2 \delta e^{-i\left(\lambda_{S}-\lambda_{L}\right) \Delta t}\right|^{2} \times D_{\mathrm{CPT}} .
\end{aligned}
$$

The expected behavior of the observables $R_{2, \mathrm{CPT}}^{\exp }(\Delta t)$ and $R_{4, \mathrm{CPT}}^{\exp }(\Delta t)$ as a function of $\Delta t$, and without the approximations of eqs. (3.15) and (3.16), is shown in figure 1, where - for visualization purposes - the probabilities involved have been evaluated fixing the CPT violating parameters $\Re \delta$ and $\Im \delta$ to a value different from zero, and equal to their present uncertainties [8], i.e. $\Re \delta=3.3 \times 10^{-4}$ and $\Im \delta=1.6 \times 10^{-5}$. In figure 2 a zoom of the $\Delta t>0$ region, where the "plateau" regimes (3.4) and (3.5) dominate, is shown. Experimentally, this is the most interesting and statistically most populated region, where the best sensitivity to CPT violation effects can be reached by the KLOE-2 experiment (see section 4).

We emphasise that these observables are genuine CPT violating effects by comparing experimentally the probability for a given transition and its CPT reverse, independent of any theoretical scenario generating this effect. When they are interpreted in a model for CPT violation in the mass matrix (i.e. with $\delta \neq 0$ ) and nothing else, these observables can be compared with the result expected for the survival probabilities (diagonal processes) like the one that has been measured by the CPLEAR experiment [18]. In this case, the 

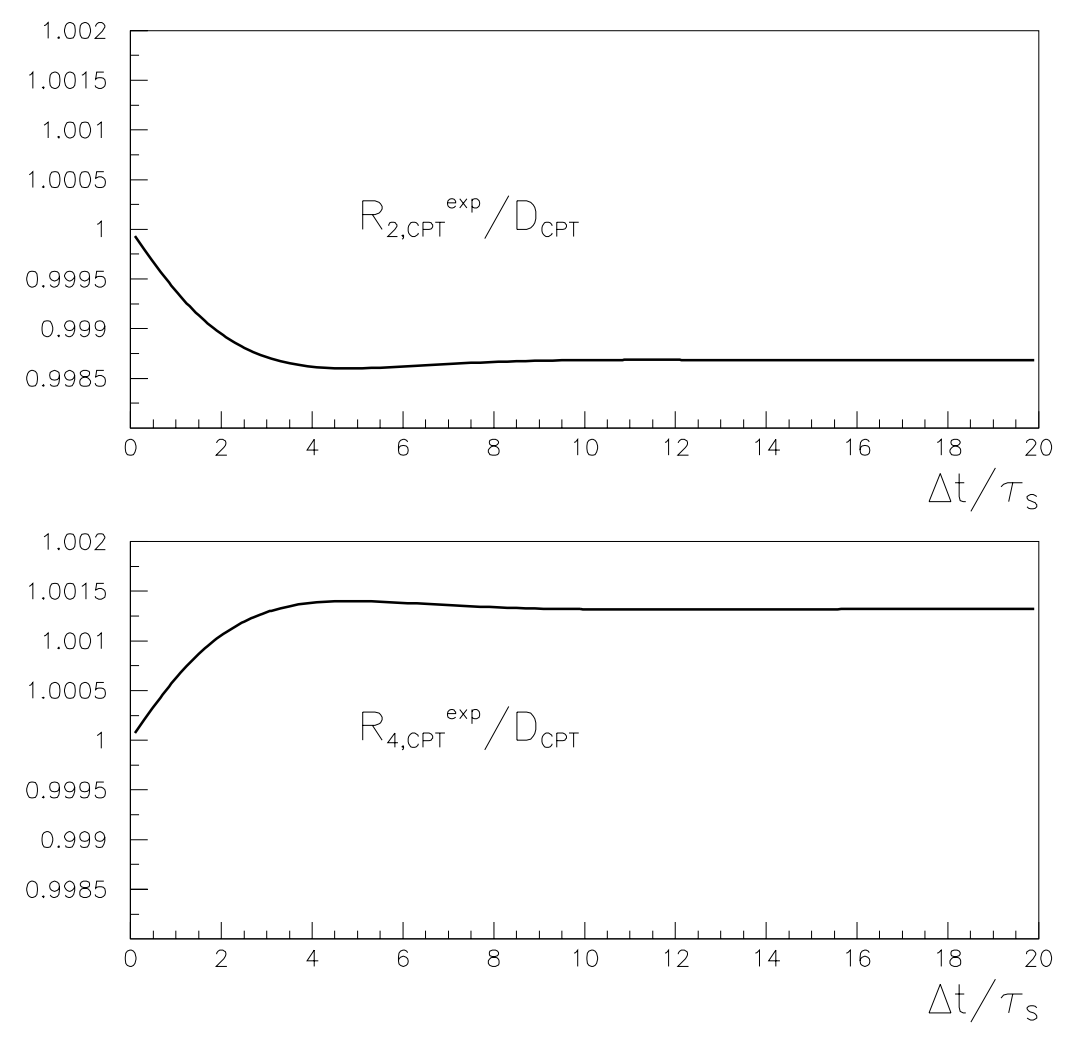

Figure 2. A zoom of the plots shown in figure 1 in the region $0 \leq \Delta t \leq 20 \tau_{S}$.

CPLEAR asymmetry can be easily translated into our formalism as an observable ratio of probabilities (in this case $D_{\mathrm{CPT}}=1$ ):

$$
\begin{aligned}
\frac{I\left(\ell^{-}, \ell^{+} ; \Delta t\right)}{I\left(\ell^{+}, \ell^{-} ; \Delta t\right)} & =\frac{P\left[\mathrm{~K}^{0}(0) \rightarrow \mathrm{K}^{0}(\Delta t)\right]}{P\left[\overline{\mathrm{K}}^{0}(0) \rightarrow \overline{\mathrm{K}}^{0}(\Delta t)\right]} \\
& \simeq|1-4 \delta|^{2}\left|1+\frac{8 \delta}{1+e^{+i\left(\lambda_{S}-\lambda_{L}\right) \Delta t}}\right|^{2}
\end{aligned}
$$

The comparison of equations (3.15) and (3.16) with equation (3.17) shows that even within the same model, the difference in the dependence on the CPT violation parameter $\delta$ is apparent. In the limit $\Delta \Gamma \rightarrow 0$ the ratio (3.17) tends to unity for all times, whereas ratios (3.15) and (3.16) are different from unity through $\Re \delta$, which is independent of $\Delta \Gamma$. Moreover, just as an illustration of the different sensitivity of these observables to CPT violation, in the hypothesis of CPT-violating effects introduced via a deviation from conventional quantum mechanics, believed to reflect the loss of quantum coherence expected in some approaches to quantum gravity [37,38], the ratio (3.17) is insensitive to these effects (up to second order in the CPT violation parameters of the model and for all times [38]), while it can be shown that ratios (3.15) and (3.16) are sensitive to them at first order in some of the parameters. 


\section{Impact of the approximations on the test. Results.}

In order to study the impact of the approximations involved in the proposed CPT test, namely negligible direct $\mathrm{CP}$ and CPT violation contributions in the $\pi \pi$ and $3 \pi^{0}$ channels, and the validity of the $\Delta S=\Delta Q$ rule, they are treated separately.

First, the effect of possible direct $\mathrm{CP}$ and $\mathrm{CPT}$ violation contributions is evaluated on the observable ratios $R_{i, \mathrm{CPT}}^{\exp }(\Delta t)$, while still assuming the $\Delta S=\Delta Q$ rule. To this aim the following parametrisation is introduced:

$$
\begin{gathered}
\eta_{\pi \pi}=\epsilon_{L}+\epsilon_{\pi \pi}^{\prime} \\
\eta_{3 \pi^{0}}=\epsilon_{S}+\epsilon_{3 \pi^{0}}^{\prime},
\end{gathered}
$$

where $\epsilon_{\pi \pi}^{\prime}$ and $\epsilon_{3 \pi^{0}}^{\prime}$ represent the generic contributions of direct CP and/or CPT violation in the $\pi \pi$ and $3 \pi^{0}$ channels, respectively. In this more general case, the orthogonality condition eqs. (2.12) is no more satisfied, and the true orthogonal pair to be considered in writing the initial state $(3.1)$ is $\left\{\mathrm{K}_{+}, \widetilde{\mathrm{K}}_{-}\right\}$(or $\left\{\widetilde{\mathrm{K}}_{+}, \mathrm{K}_{-}\right\}$) instead of $\left\{\mathrm{K}_{+}, \mathrm{K}_{-}\right\}$. The effect of $\epsilon_{\pi \pi}^{\prime}$ and $\epsilon_{3 \pi^{0}}^{\prime}$ can be easily singled out in the explicit expressions of the observable ratios (neglecting higher order terms in small parameters and for not too large negative $\Delta t$ ):

$$
\begin{aligned}
R_{2, \mathrm{CPT}}^{\exp }(\Delta t) & =\frac{P\left[\mathrm{~K}^{0}(0) \rightarrow \mathrm{K}_{-}(\Delta t)\right]}{P\left[\widetilde{\mathrm{K}}_{-}(0) \rightarrow \overline{\mathrm{K}}^{0}(\Delta t)\right]} \times D_{\mathrm{CPT}} \\
& =\frac{\left|e^{-i \lambda_{S} \Delta t}\left(\frac{1-\epsilon_{L}}{\sqrt{2}}\right) \eta_{3 \pi^{0}}+e^{-i \lambda_{L} \Delta t}\left(\frac{1-\epsilon_{S}}{\sqrt{2}}\right)\right|^{2}}{\left|e^{-i \lambda_{S} \Delta t}\left(\frac{1-\epsilon_{S}}{\sqrt{2}}\right) \eta_{\pi \pi}+e^{-i \lambda_{L} \Delta t}\left(\frac{1-\epsilon_{L}}{\sqrt{2}}\right)\right|^{2}} \times D_{\mathrm{CPT}}, \\
& \simeq|1-2 \delta|^{2}\left|1+\left(\eta_{3 \pi^{0}}-\eta_{\pi \pi}\right) e^{-i\left(\lambda_{S}-\lambda_{L}\right) \Delta t}\right|^{2} \times D_{\mathrm{CPT}}, \\
& =|1-2 \delta|^{2}\left|1+\left(2 \delta+\epsilon_{3 \pi^{0}}^{\prime}-\epsilon_{\pi \pi}^{\prime}\right) e^{-i\left(\lambda_{S}-\lambda_{L}\right) \Delta t}\right|^{2} \times D_{\mathrm{CPT}}, \\
R_{4, \mathrm{CPT}}^{\exp }(\Delta t) & =\frac{P\left[\overline{\mathrm{K}}^{0}(0) \rightarrow \mathrm{K}_{-}(\Delta t)\right]}{P\left[\widetilde{\mathrm{K}}_{-}(0) \rightarrow \mathrm{K}^{0}(\Delta t)\right]} \times D_{\mathrm{CPT}} \\
& =\frac{\left|e^{-i \lambda_{S} \Delta t}\left(\frac{1+\epsilon_{L}}{\sqrt{2}}\right) \eta_{3 \pi^{0}}-e^{-i \lambda_{L} \Delta t}\left(\frac{1+\epsilon_{S}}{\sqrt{2}}\right)\right|^{2}}{\left|e^{-i \lambda_{S} \Delta t}\left(\frac{1+\epsilon_{S}}{\sqrt{2}}\right) \eta_{\pi \pi}-e^{-i \lambda_{L} \Delta t}\left(\frac{1+\epsilon_{L}}{\sqrt{2}}\right)\right|^{2} \times D_{\mathrm{CPT}}} \\
& \simeq|1+2 \delta|^{2}\left|1-\left(\eta_{3 \pi^{0}}-\eta_{\pi \pi}\right) e^{-i\left(\lambda_{S}-\lambda_{L}\right) \Delta t}\right|^{2} \times D_{\mathrm{CPT}}, \\
& =|1+2 \delta|^{2}\left|1-\left(2 \delta+\epsilon_{3 \pi^{0}}^{\prime}-\epsilon_{\pi \pi}^{\prime}\right) e^{-i\left(\lambda_{S}-\lambda_{L}\right) \Delta t}\right|^{2} \times D_{\mathrm{CPT}} .
\end{aligned}
$$

It is important to realise from eqs. (4.2) and (4.3) that there exists a sum rule for $\Delta t \gtrsim 0$ given by:

$$
R_{2, \mathrm{CPT}}^{\exp }(\Delta t)+R_{4, \mathrm{CPT}}^{\exp }(\Delta t)=2 D_{\mathrm{CPT}}
$$

indicating that the quantity $D_{\mathrm{CPT}}$ is measurable within the same experiment. 
For $\Delta t=0$ the deviation of each ratio from unity (once $D_{\mathrm{CPT}}$ is factored out) is only given by the contaminating parameters $\Re \epsilon_{3 \pi^{0}}^{\prime}$ and $\Re \epsilon_{\pi \pi}^{\prime}$ :

$$
\begin{aligned}
& R_{2, \mathrm{CPT}}^{\exp }(0)=\left[1+2 \Re\left(\epsilon_{3 \pi^{0}}^{\prime}-\epsilon_{\pi \pi}^{\prime}\right)\right] \times D_{\mathrm{CPT}} \\
& R_{4, \mathrm{CPT}}^{\exp }(0)=\left[1-2 \Re\left(\epsilon_{3 \pi^{0}}^{\prime}-\epsilon_{\pi \pi}^{\prime}\right)\right] \times D_{\mathrm{CPT}} .
\end{aligned}
$$

In the limit $\Delta t \gg \tau_{S}$ there is no dependence on $\eta_{3 \pi^{0}}$ or $\eta_{\pi \pi}$, the limits (3.4) and (3.5) are fully recovered:

$$
\begin{aligned}
& R_{2, \mathrm{CPT}}^{\exp }\left(\Delta t \gg \tau_{S}\right)=(1-4 \Re \delta) \times D_{\mathrm{CPT}} \\
& R_{4, \mathrm{CPT}}^{\exp }\left(\Delta t \gg \tau_{S}\right)=(1+4 \Re \delta) \times D_{\mathrm{CPT}},
\end{aligned}
$$

and any deviation from unity would be a very clean and unambiguous signal of CPT violation, once $D_{\mathrm{CPT}}$ is factored out.

The presence of direct $\mathrm{CP}$ violation contributions can mimic CPT violation effects mostly in the $\Delta t<0$ region, while for $\Delta t \gg \tau_{S}$ it does not affect the observable ratios (see eqs. (4.7) and (4.8)), as it can be seen in the plots of figures 3 and 4 . In these plots the fake effects have been amplified with unrealistic values of $\eta_{3 \pi^{0}}^{\prime}$ in order to visualise them, ${ }^{3}$ varying of $\pm 10 \%$ the absolute value of $\eta_{3 \pi^{0}}$, or of $\pm 10^{\circ}$ its phase, with respect to the value $\eta_{3 \pi^{0}}=\epsilon_{S}$.

From these plots one also realises that the time dependences induced by $\delta$ and $\epsilon_{3 \pi^{0}}^{\prime}$ (or $\left.\epsilon_{\pi \pi}^{\prime}\right)$, mostly in the $\Delta t<0$ region, are different and therefore these parameters could be disentangled, at least in principle. One should also consider that possible contributions from direct CPT violation in the decay amplitudes in the $\epsilon_{3 \pi^{0}}^{\prime}$ and $\epsilon_{\pi \pi}^{\prime}$ parameters, cannot be considered fake effects, while instead they are genuine CPT violating effects, e.g. responsible of making the observable ratios (4.5) and (4.6) different from unity at $\Delta t=0$.

As a conclusion the effect of the contamination due to direct $\mathrm{CP}$ violation can be considered fully controllable, and totally irrelevant in the limits (4.7) and (4.8).

If also the $\Delta S=\Delta Q$ rule is not assumed, in analogy with the formalism for the $\left|\mathrm{K}_{+}\right\rangle$ and $\left|\mathrm{K}_{-}\right\rangle$states introduced in section 2, one should introduce the tagged states which cannot decay into $\ell^{-}$or $\ell^{+}$, respectively, as:

$$
\begin{aligned}
\left|\widetilde{\mathrm{K}}_{0}\right\rangle & \equiv \widetilde{\mathrm{N}}_{0}\left[\left|\mathrm{~K}_{\mathrm{L}}\right\rangle-\eta_{\ell^{-}}\left|\mathrm{K}_{\mathrm{S}}\right\rangle\right] \\
\left|\widetilde{\mathrm{K}}_{\overline{0}}\right\rangle & \equiv \widetilde{\mathrm{N}}_{\overline{0}}\left[\left|\mathrm{~K}_{\mathrm{L}}\right\rangle-\eta_{\ell^{+}}\left|\mathrm{K}_{\mathrm{S}}\right\rangle\right]
\end{aligned}
$$

with $\widetilde{\mathrm{N}}_{0}, \widetilde{\mathrm{N}}_{\overline{0}}$ two suitable normalization factors,

$$
\begin{aligned}
& \eta_{\ell^{+}}=\frac{\left\langle\ell^{+}|T| \mathrm{K}_{\mathrm{L}}\right\rangle}{\left\langle\ell^{+}|T| \mathrm{K}_{\mathrm{S}}\right\rangle}=1-2 \delta-2 x_{+}-2 x_{-} \\
& \eta_{\ell^{-}}=\frac{\left\langle\ell^{-}|T| \mathrm{K}_{\mathrm{L}}\right\rangle}{\left\langle\ell^{-}|T| \mathrm{K}_{\mathrm{S}}\right\rangle}=-1-2 \delta+2 x_{+}^{\star}-2 x_{-}^{\star},
\end{aligned}
$$

\footnotetext{
${ }^{3}$ At lowest order in Chiral Perturbation Theory one has [39-41] that $\epsilon_{3 \pi^{0}}^{\prime}=-2 \epsilon^{\prime}$, with $\epsilon^{\prime}$ the direct $\mathrm{CP}$ violation parameter in $\pi \pi$ decays. Therefore the direct $\mathrm{CP}$ violation contribution is expected to be negligible. The experimental knowledge on the $\eta_{3 \pi^{0}}$ parameter is much less precise than for $\eta_{\pi \pi}$, resulting at present in an upper limit: $\left|\eta_{3 \pi^{0}}\right|<8.8 \times 10^{-3}$ at $90 \%$ C.L. [42]. In the plots of figures 3 and 4 we considered the contribution of $\epsilon_{3 \pi^{0}}^{\prime}$ enhanced in magnitude by a large safety factor (of order one hundred) with respect to the Chiral Perturbation Theory prediction.
} 

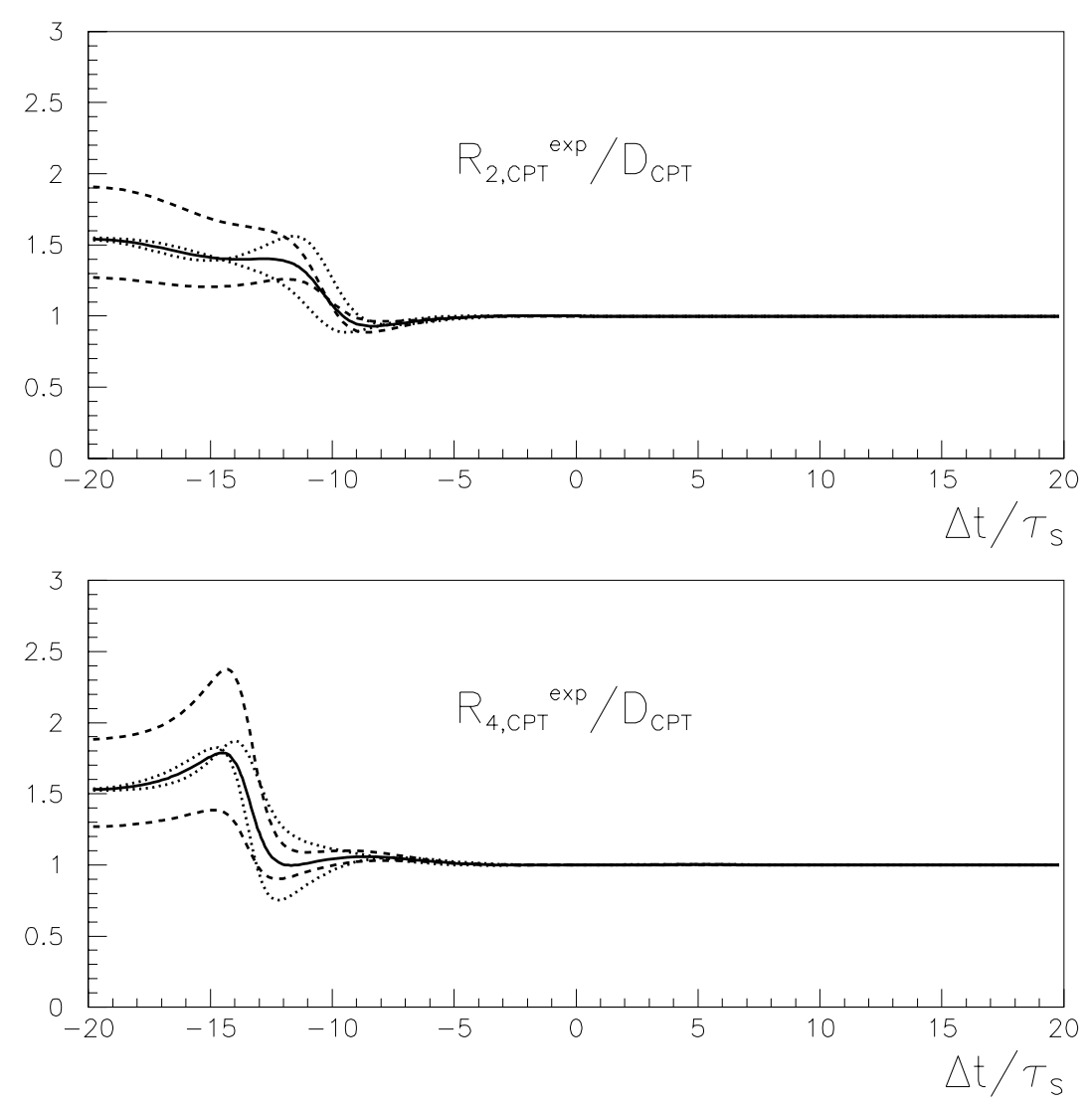

Figure 3. The expected ratios for the CPT test $R_{2, \mathrm{CPT}}^{\exp }(\Delta t)$ (top) and $R_{4, \mathrm{CPT}}^{\exp }(\Delta t)$ (bottom) as a function of $\Delta t$ (solid line); dashed lines correspond to $\pm 10 \%$ variation in the absolute value of $\eta_{3 \pi^{0}}$, while dotted lines correspond to a $\pm 10^{\circ}$ variation of its phase (with respect to the value $\left.\eta_{3 \pi^{0}}=\epsilon_{S}\right)$. For visualization purposes the $\mathrm{CPT}$ violating parameters have been fixed to the values $\Re \delta=3.3 \times 10^{-4}$ and $\Im \delta=1.6 \times 10^{-5}$.

and the $x_{+}, x_{-}$parameters defined as:

$$
x_{ \pm}=\frac{1}{2}\left[\left(\frac{A\left(\overline{\mathrm{K}}^{0} \rightarrow \ell^{+}\right)}{A\left(\mathrm{~K}^{0} \rightarrow \ell^{+}\right)}\right) \pm\left(\frac{A\left(\mathrm{~K}^{0} \rightarrow \ell^{-}\right)}{A\left(\overline{\mathrm{K}}^{0} \rightarrow \ell^{-}\right)}\right)^{\star}\right]
$$

and corresponding to CPT-invariant and CPT-violating $\Delta S=\Delta Q$ rule violation, respectively.

The orthogonal partners of $\left|\widetilde{\mathrm{K}}_{0}\right\rangle$ and $\left|\widetilde{\mathrm{K}}_{\overline{0}}\right\rangle$ states are, respectively:

$$
\begin{aligned}
\left|\mathrm{K}_{\overline{0}}\right\rangle & \equiv \mathrm{N}_{\overline{0}}\left[\left|\mathrm{~K}_{\mathrm{L}}\right\rangle+\gamma_{-}\left|\mathrm{K}_{\mathrm{S}}\right\rangle\right] \\
\left|\mathrm{K}_{0}\right\rangle & \equiv \mathrm{N}_{0}\left[\left|\mathrm{~K}_{\mathrm{L}}\right\rangle+\gamma_{+}\left|\mathrm{K}_{\mathrm{S}}\right\rangle\right]
\end{aligned}
$$

with

$$
\gamma_{ \pm}=\frac{1-\eta_{\ell_{ \pm}}^{\star}\left\langle\mathrm{K}_{\mathrm{S}} \mid \mathrm{K}_{\mathrm{L}}\right\rangle}{\eta_{\ell_{ \pm}}^{\star}-\left\langle\mathrm{K}_{\mathrm{L}} \mid \mathrm{K}_{\mathrm{S}}\right\rangle}
$$



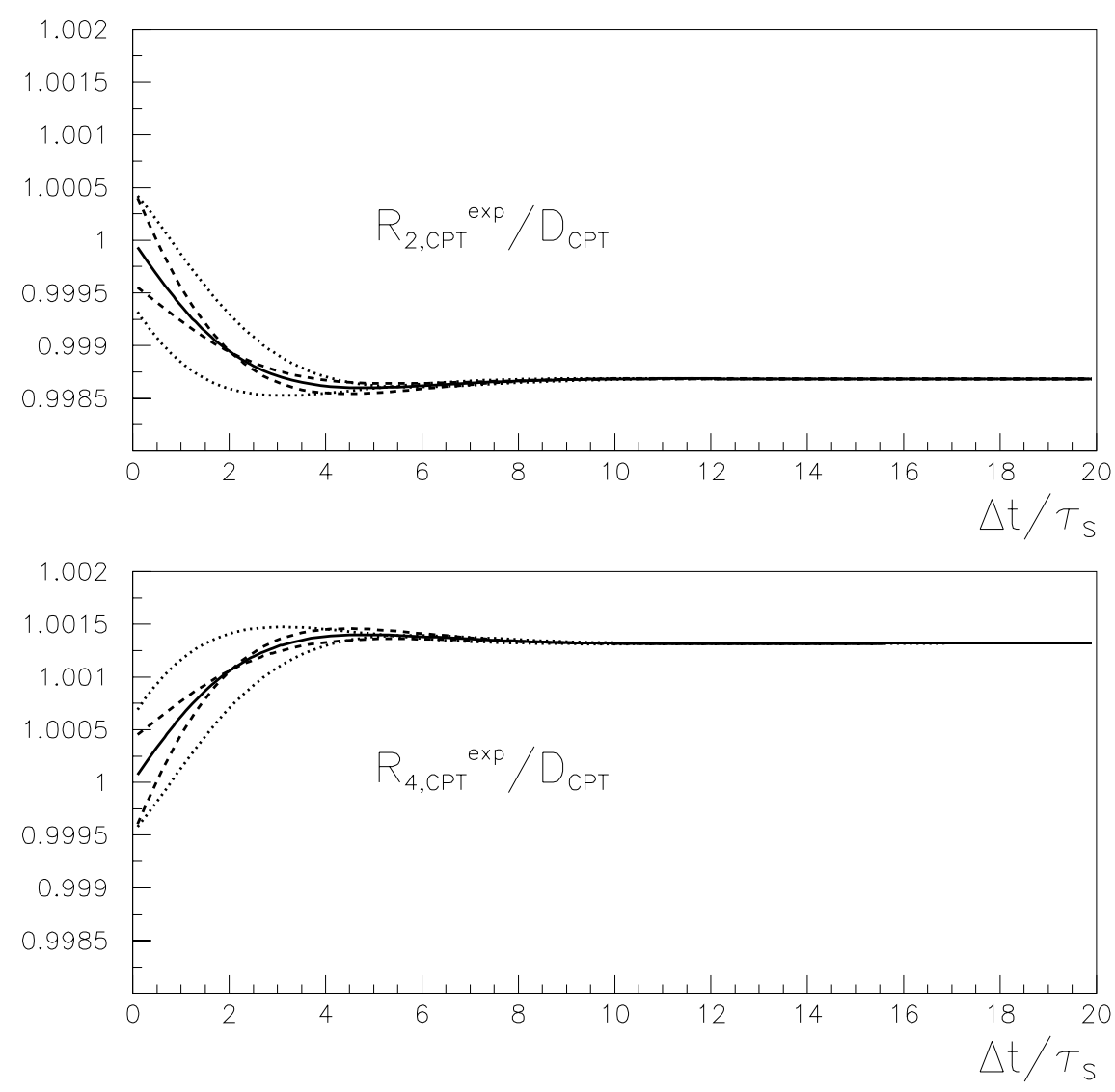

Figure 4. A zoom of the plots shown in figure 3 in the region $0 \leq \Delta t \leq 20 \tau_{S}$.

and $\mathrm{N}_{0}, \mathrm{~N}_{\overline{0}}$ two suitable normalization factors.

The orthogonal pairs $\left\{\widetilde{\mathrm{K}}_{0}, \mathrm{~K}_{\overline{0}}\right\}$ and $\left\{\widetilde{\mathrm{K}}_{\overline{0}}, \mathrm{~K}_{0}\right\}$ (note the different symbols adopted in this case with respect to $\mathrm{K}^{0}, \overline{\mathrm{K}}^{0}$ ) constitute now the true orthogonal bases to be considered. The effect of the $\Delta S \neq \Delta Q$ parameters $x_{+}$and $x_{-}$can be easily singled out in the explicit expressions of the observable ratios (still neglecting higher order terms in small parameters and for not too large negative $\Delta t$ ):

$$
\begin{aligned}
R_{2, \mathrm{CPT}}^{\exp }(\Delta t) & =\frac{P\left[\widetilde{\mathrm{K}}_{0}(0) \rightarrow \mathrm{K}_{-}(\Delta t)\right]}{P\left[\widetilde{\mathrm{K}}_{-}(0) \rightarrow \mathrm{K}_{\overline{0}}(\Delta t)\right]} \times D_{\mathrm{CPT}} \\
& =\frac{\left|e^{-i \lambda_{S} \Delta t}\left(\eta_{\ell^{-}}\right)\left(\eta_{3 \pi^{0}}\right)-e^{-i \lambda_{L} \Delta t}\right|^{2}}{\left|e^{-i \lambda_{S} \Delta t}\left(\eta_{\pi \pi}\right)-e^{-i \lambda_{L} \Delta t}\left(\eta_{\ell^{-}}\right)\right|^{2}} \times D_{\mathrm{CPT}}, \\
& \simeq\left|\frac{1}{\eta_{\ell^{-}}}\right|^{2}\left|1+\left(\frac{\eta_{\pi \pi}}{\eta_{\ell^{-}}}-\left(\eta_{\ell^{-}}\right)\left(\eta_{3 \pi^{0}}\right)\right) e^{-i\left(\lambda_{S}-\lambda_{L}\right) \Delta t}\right|^{2} \times D_{\mathrm{CPT}}, \\
& \simeq\left|1-2 \delta+2 x_{+}^{\star}-2 x_{-}^{\star}\right|^{2}\left|1+\left(\eta_{3 \pi^{0}}-\eta_{\pi \pi}\right) e^{-i\left(\lambda_{S}-\lambda_{L}\right) \Delta t}\right|^{2} \times D_{\mathrm{CPT}}, \\
& =\left|1-2 \delta+2 x_{+}^{\star}-2 x_{-}^{\star}\right|^{2}\left|1+\left(2 \delta+\epsilon_{3 \pi^{0}}^{\prime}-\epsilon_{\pi \pi}^{\prime}\right) e^{-i\left(\lambda_{S}-\lambda_{L}\right) \Delta t}\right|^{2} \times D_{\mathrm{CPT}},
\end{aligned}
$$




$$
\begin{aligned}
R_{4, \mathrm{CPT}}^{\exp }(\Delta t) & =\frac{P\left[\widetilde{\mathrm{K}}_{\overline{0}}(0) \rightarrow \mathrm{K}_{-}(\Delta t)\right]}{P\left[\widetilde{\mathrm{K}}_{-}(0) \rightarrow \mathrm{K}_{0}(\Delta t)\right]} \times D_{\mathrm{CPT}} \\
& =\frac{\left|e^{-i \lambda_{S} \Delta t}\left(\eta_{\ell^{+}}\right)\left(\eta_{3 \pi^{0}}\right)-e^{-i \lambda_{L} \Delta t}\right|^{2}}{\left|e^{-i \lambda_{S} \Delta t}\left(\eta_{\pi \pi}\right)-e^{-i \lambda_{L} \Delta t}\left(\eta_{\ell^{+}}\right)\right|^{2}} \times D_{\mathrm{CPT}}, \\
& \simeq\left|\frac{1}{\eta_{\ell^{+}}}\right|^{2}\left|1+\left(\frac{\eta_{\pi \pi}}{\eta_{\ell^{+}}}-\left(\eta_{\ell^{+}}\right)\left(\eta_{3 \pi^{0}}\right)\right) e^{-i\left(\lambda_{S}-\lambda_{L}\right) \Delta t}\right|^{2} \times D_{\mathrm{CPT}}, \\
& \simeq\left|1+2 \delta+2 x_{+}+2 x_{-}\right|^{2}\left|1-\left(\eta_{3 \pi^{0}}-\eta_{\pi \pi}\right) e^{-i\left(\lambda_{S}-\lambda_{L}\right) \Delta t}\right|^{2} \times D_{\mathrm{CPT}} \\
& =\left|1+2 \delta+2 x_{+}+2 x_{-}\right|^{2}\left|1-\left(2 \delta+\epsilon_{3 \pi^{0}}^{\prime}-\epsilon_{\pi \pi}^{\prime}\right) e^{-i\left(\lambda_{S}-\lambda_{L}\right) \Delta t}\right|^{2} \times D_{\mathrm{CPT}},
\end{aligned}
$$

In the limit $\Delta t=0$ the deviation of each ratio from unity (once $D_{\mathrm{CPT}}$ is factored out) is given by the contaminating parameters $\Re \epsilon_{3 \pi^{0}}^{\prime}, \Re \epsilon_{\pi \pi}^{\prime}$, and $x_{+}$, and by the $x_{-}$parameter, which is explicitly CPT violating in the $\Delta S \neq \Delta Q$ decay amplitudes, and can be considered a genuine source of CPT violation:

$$
\begin{aligned}
& R_{2, \mathrm{CPT}}^{\exp }(0)=\left[1+2 \Re\left(\epsilon_{3 \pi^{0}}^{\prime}-\epsilon_{\pi \pi}^{\prime}\right)+4 \Re\left(x_{+}-x_{-}\right)\right] \times D_{\mathrm{CPT}} \\
& R_{4, \mathrm{CPT}}^{\exp }(0)=\left[1-2 \Re\left(\epsilon_{3 \pi^{0}}^{\prime}-\epsilon_{\pi \pi}^{\prime}\right)+4 \Re\left(x_{+}+x_{-}\right)\right] \times D_{\mathrm{CPT}}
\end{aligned}
$$

In the limit $\Delta t \gg \tau_{S}$ we get:

$$
\begin{aligned}
& R_{2, \mathrm{CPT}}^{\exp }\left(\Delta t \gg \tau_{S}\right)=\left(1-4 \Re \delta+4 \Re x_{+}-4 \Re x_{-}\right) \times D_{\mathrm{CPT}} \\
& R_{4, \mathrm{CPT}}^{\exp }\left(\Delta t \gg \tau_{S}\right)=\left(1+4 \Re \delta+4 \Re x_{+}+4 \Re x_{-}\right) \times D_{\mathrm{CPT}}
\end{aligned}
$$

These results suggest the possibility of having a measurement independent of $x_{+}$and $D_{\mathrm{CPT}}$, directly measuring the double ratio:

$$
\begin{aligned}
\frac{R_{2, \mathrm{CPT}}^{\exp }(\Delta t)}{R_{4, \mathrm{CPT}}^{\exp }(\Delta t)} & \simeq\left(1-8 \Re \delta-8 \Re x_{-}\right)\left|1+2\left(\eta_{3 \pi^{0}}-\eta_{\pi \pi}\right) e^{-i\left(\lambda_{S}-\lambda_{L}\right) \Delta t}\right|^{2} \\
& =\left(1-8 \Re \delta-8 \Re x_{-}\right)\left|1+2\left(2 \delta+\epsilon_{3 \pi^{0}}^{\prime}-\epsilon_{\pi \pi}^{\prime}\right) e^{-i\left(\lambda_{S}-\lambda_{L}\right) \Delta t}\right|^{2}
\end{aligned}
$$

which becomes for $\Delta t=0$ :

$$
\frac{R_{2, \mathrm{CPT}}^{\exp }(0)}{R_{4, \mathrm{CPT}}^{\exp }(0)}=1-8 \Re x_{-}+4 \Re\left(\epsilon_{3 \pi^{0}}^{\prime}-\epsilon_{\pi \pi}^{\prime}\right) .
$$

and in the limit $\Delta t \gg \tau_{S}$ :

$$
\frac{R_{2, \mathrm{CPT}}^{\exp }\left(\Delta t \gg \tau_{S}\right)}{R_{4, \mathrm{CPT}}^{\exp }\left(\Delta t \gg \tau_{S}\right)}=1-8 \Re \delta-8 \Re x_{-} .
$$

The double ratio (4.23) constitutes one of the most robust observables for our proposed CPT test. In the limit $\Delta t \gg \tau_{S}$ it exhibits a pure and genuine CPT violating effect, 
even without the assumptions of the validity of the $\Delta S=\Delta Q$ rule and of negligible contaminations from direct $\mathrm{CP}$ violation.

In principle, the possible contribution of $\Re x_{-}$might be disentangled from the one of $\Re \delta$, or at least bound, by making a study of the time dependence of the double ratio in the $\Delta t<0$ region - the most difficult experimentally, due to the lack of statistics — and independently measuring the direct $\mathrm{CP}$ violation contributions in $3 \pi^{0}$ and $\pi \pi$ decays, or making an ansatz on their size.

The KLOE-2 experiment at the DA $\Phi$ NE facility could make a measurement of the two observable ratios $R_{2, \mathrm{CPT}}^{\exp }(\Delta t)$ and $R_{4, \mathrm{CPT}}^{\exp }(\Delta t)$, with an integrated luminosity $L$ of $\mathcal{O}\left(10 \mathrm{fb}^{-1}\right)$ [28]. The $I\left(f_{1}, f_{2} ; \Delta t\right)$ distributions have been evaluated with a simple Monte Carlo simulation, making the approximation of a gaussian $\Delta t$ experimental resolution with $\sigma=1 \tau_{S}$, and a full detection efficiency, as discussed in detail in ref. [22]. From this study it emerges that the $I\left(\ell^{ \pm}, 3 \pi^{0} ; \Delta t\right)$ distributions, at the considered integrated luminosity, have very few or no events for $\Delta t \lesssim-5 \tau_{S}$. While a complete feasibility study is beyond the scope of the present paper, by considering a large $\Delta t$ interval in the statistically most populated region, e.g. $0 \leq \Delta t \leq 300 \tau_{S}$, which is the most interesting one for our CPT test, a statistical sensitivity on the double ratio $(4.25)$ of $\left(3.0 \times 10^{-3}\right),\left(2.1 \times 10^{-3}\right)$, and $\left(1.5 \times 10^{-3}\right)$ is obtained for $L=5,10$, and $20 \mathrm{fb}^{-1}$, respectively. Once translated into an uncertainty on $\Re \delta$, these results might improve the present best measurement by CPLEAR [18].

As a final remark it's worth noting that the double ratio in eq. (4.25) in practice corresponds to the following ratio of combinations of the semileptonic asymmetries for $\mathrm{K}_{\mathrm{S}}$ and $\mathrm{K}_{\mathrm{L}}$ :

$$
\frac{R_{2, \mathrm{CPT}}^{\exp }\left(\Delta t \gg \tau_{S}\right)}{R_{4, \mathrm{CPT}}^{\exp }\left(\Delta t \gg \tau_{S}\right)}=\frac{1+A_{L}}{1-A_{L}} \times \frac{1-A_{S}}{1+A_{S}} \simeq 1+2\left(A_{L}-A_{S}\right)
$$

with

$$
\begin{aligned}
& A_{S}=\frac{\Gamma\left(\mathrm{K}_{\mathrm{S}} \rightarrow \ell^{+}\right)-\Gamma\left(\mathrm{K}_{\mathrm{S}} \rightarrow \ell^{-}\right)}{\Gamma\left(\mathrm{K}_{\mathrm{S}} \rightarrow \ell^{+}\right)+\Gamma\left(\mathrm{K}_{\mathrm{S}} \rightarrow \ell^{-}\right)} \\
& A_{L}=\frac{\Gamma\left(\mathrm{K}_{\mathrm{L}} \rightarrow \ell^{+}\right)-\Gamma\left(\mathrm{K}_{\mathrm{L}} \rightarrow \ell^{-}\right)}{\Gamma\left(\mathrm{K}_{\mathrm{L}} \rightarrow \ell^{+}\right)+\Gamma\left(\mathrm{K}_{\mathrm{L}} \rightarrow \ell^{-}\right)} .
\end{aligned}
$$

Eq. (4.26) is model independent in the interpretation of the CPT-violating asymmetries. Therefore the same observable could be accessible by measuring separately and independently $A_{S}$ and $A_{L}$, instead of the double ratio at large $\Delta t$ (4.25). This is indeed a part of the KLOE-2 program [28].

\section{Conclusions}

A novel CPT test has been studied in the neutral kaon system based on the direct comparison of a transition probability with its CPT reverse transition. The appropriate preparation and detection of in and out states in both the reference and the reverse processes is made by exploiting the EPR entanglement of neutral kaons produced in a $\phi$-factory and using their decays as filtering measurements of the kaon states only. 
We have built the time dependence of two independent ratios of observable quantities, which relate to a given transition and to its CPT-transformed, by selecting the two semileptonic decays for flavour tag, the $\pi \pi$ and $3 \pi^{0}$ decays for CP tag, and the two time orderings of the decay pairs. These observables have been interpreted in the standard Weisskopf-Wigner approach by means of the CPT violating $\delta$ parameter in the mass matrix of $\mathrm{K}^{0}-\overline{\mathrm{K}}^{0}$. The dynamical CPT-asymmetry is generated with the time evolution, the observable ratios being unity at $\Delta t=0$, and for $\Delta t \gg \tau_{S}$ beyond the interference region, where one has the best statistics of events, the deviation from unity is given by $\Re \delta$, a genuine CPT violating parameter independent of $\Delta \Gamma$, for which the decay is not an essential ingredient.

We have demonstrated that the necessary knowledge of the decay properties is controllable and some strategies, like a sum rule and the ratio of the observable ratios, can be used. Furthermore possible spurious effects induced by CP violation in the decay and/or a violation of the $\Delta S=\Delta Q$ rule have been shown to be either negligible or disentangled by the dependence with the time evolution. The proposed measurement is thus fully robust leading to definite conclusions on CPT Violation.

These observables would be able to be measured in the KLOE-2 experiment at the $D A \Phi N E$ facility in Frascati, with a statistical precision of $\mathcal{O}\left(10^{-3}\right)$.

\section{Acknowledgments}

This research has been supported by MINECO and Generalitat Valenciana Projects FPA 2011-23596 and GVPROMETEO II 2013-017 and by Severo Ochoa Excellence Centre Project SEV 2014-0398.

Open Access. This article is distributed under the terms of the Creative Commons Attribution License (CC-BY 4.0), which permits any use, distribution and reproduction in any medium, provided the original author(s) and source are credited.

\section{References}

[1] G. Lüders, Proof of the TCP theorem, Annals Phys. 2 (1957) 1 [INSPIRE].

[2] W. Pauli, Exclusion principle, Lorentz group and reflexion of space-time and charge, in Niels Bohr and the development of physics, edited by W. Pauli, Pergamon, London (1955).

[3] J.S. Bell, Time reversal in field theory, Proc. R. Soc. London A 231 (1955) 479.

[4] R. Jost, A remark on the C.T.P. theorem, Helv. Phys. Acta 30 (1957) 409 [InSPIRE].

[5] O.W. Greenberg, CPT violation implies violation of Lorentz invariance, Phys. Rev. Lett. 89 (2002) 231602 [hep-ph/0201258] [INSPIRE].

[6] O.W. Greenberg, Why is CPT fundamental?, Found. Phys. 36 (2006) 1535 [hep-ph/0309309] [INSPIRE].

[7] S. Hollands, A General PCT theorem for the operator product expansion in curved space-time, Commun. Math. Phys. 244 (2004) 209 [gr-qc/0212028] [INSPIRE]. 
[8] Particle Data Group collaboration, K.A. Olive et al., Review of Particle Physics, Chin. Phys. C 38 (2014) 090001 [inSPIRE].

[9] KLOE collaboration, F. Ambrosino et al., First observation of quantum interference in the process $\phi \rightarrow K_{S} K_{L} \rightarrow \pi^{+} \pi^{-} \pi^{+} \pi^{-}$: A Test of quantum mechanics and CPT symmetry, Phys. Lett. B 642 (2006) 315 [hep-ex/0607027] [INSPIRE].

[10] KLOE collaboration, A. Di Domenico et al., Search for CPT violation and decoherence effects in the neutral kaon system, J. Phys. Conf. Ser. 171 (2009) 012008 [InSPIRE].

[11] KLOE collaboration, A. Di Domenico et al., CPT symmetry and quantum mechanics tests in the neutral kaon system at KLOE, Found. Phys. 40 (2010) 852 [InSPIRE].

[12] KLOE-2 collaboration, D. Babusci et al., Test of CPT and Lorentz symmetry in entangled neutral kaons with the KLOE experiment, Phys. Lett. B 730 (2014) 89 [arXiv:1312.6818] [INSPIRE].

[13] CPLEAR collaboration, R. Adler et al., Tests of CPT symmetry and quantum mechanics with experimental data from CPLEAR, Phys. Lett. B 364 (1995) 239 [hep-ex/9511001] [INSPIRE].

[14] V. Weisskopf and E.P. Wigner, Calculation of the natural brightness of spectral lines on the basis of Dirac's theory, Z. Phys. 63 (1930) 54 [INSPIRE].

[15] P.K. Kabir, The CP puzzle, Academic Press, London U.K. (1968).

[16] O. Nachtmann, Elementary Particle Physics: Concepts and Phenomena, Springer-Verlag, Berlin Germany (1990).

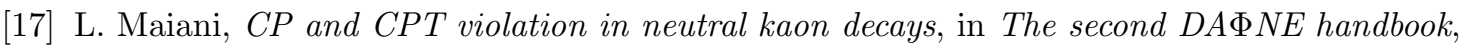
ed. L. Maiani, G. Pancheri, N. Paver, Vol.I p.3-26, INFN-LNF, Frascati (1995).

[18] CPLEAR collaboration, A. Angelopoulos et al., A Determination of the CPT violation parameter Re(delta) from the semileptonic decay of strangeness tagged neutral kaons, Phys. Lett. B 444 (1998) 52 [INSPIRE].

[19] J.S. Bell and J. Steinberger, Weak interactions of kaons, Proc. of the Oxford Int. Conf. on Elementary Particles (1965).

[20] KLOE collaboration, F. Ambrosino et al., Determination of CP and CPT violation parameters in the neutral kaon system using the Bell-Steinberger relation and data from the KLOE experiment, JHEP 12 (2006) 011 [hep-ex/0610034] [INSPIRE].

[21] J. Bernabeu and F. Martinez-Vidal, Colloquium: Time-reversal violation with quantum-entangled B mesons, Rev. Mod. Phys. 87 (2015) 165 [arXiv:1410.1742] [INSPIRE].

[22] J. Bernabeu, A. Di Domenico and P. Villanueva-Perez, Direct test of time reversal symmetry in the entangled neutral kaon system at a $\phi$-factory, Nucl. Phys. B 868 (2013) 102.

[23] M.C. Banuls and J. Bernabeu, CP, T and CPT versus temporal asymmetries for entangled states of the $B_{d}$ system, Phys. Lett. B 464 (1999) 117 [hep-ph/9908353] [INSPIRE].

[24] M.C. Banuls and J. Bernabeu, Studying indirect violation of CP, T and CPT in a B factory, Nucl. Phys. B 590 (2000) 19 [hep-ph/0005323] [INSPIRE].

[25] J. Bernabeu, F. Martinez-Vidal and P. Villanueva-Perez, Time Reversal Violation from the entangled B0-antiB0 system, JHEP 08 (2012) 064 [arXiv: 1203.0171] [INSPIRE].

[26] BABAR collaboration, J.P. Lees et al., Observation of Time Reversal Violation in the $B^{0}$ Meson System, Phys. Rev. Lett. 109 (2012) 211801 [arXiv:1207.5832] [InSPIRE]. 
[27] L. Wolfenstein, The search for direct evidence for time reversal violation, Int. J. Mod. Phys. E 8 (1999) 501 [INSPIRE].

[28] G. Amelino-Camelia et al., Physics with the KLOE-2 experiment at the upgraded DA $\Phi N E$, Eur. Phys. J. C 68 (2010) 619 [arXiv:1003.3868] [inSPIRE].

[29] T.T. Wu and C.-N. Yang, Phenomenological Analysis of Violation of CP Invariance in Decay of K0 and anti-K0, Phys. Rev. Lett. 13 (1964) 380 [INSPIRE].

[30] E. Applebaum, A. Efrati, Y. Grossman, Y. Nir and Y. Soreq, Subtleties in the BABAR measurement of time-reversal violation, Phys. Rev. D 89 (2014) 076011 [arXiv:1312.4164] [INSPIRE].

[31] J. Bernabeu, F.J. Botella and M. Nebot, Novel T-Violation observable open to any pair of decay channels at meson factories, Phys. Lett. B 728 (2014) 95 [arXiv:1309.0439] [INSPIRE].

[32] A. Einstein, B. Podolsky and N. Rosen, Can quantum mechanical description of physical reality be considered complete?, Phys. Rev. 47 (1935) 777 [INSPIRE].

[33] J. Bernabeu, N.E. Mavromatos and J. Papavassiliou, Novel type of CPT violation for correlated EPR states, Phys. Rev. Lett. 92 (2004) 131601 [hep-ph/0310180] [INSPIRE].

[34] J. Bernabeu, N.E. Mavromatos, J. Papavassiliou and A. Waldron-Lauda, Intrinsic CPT violation and decoherence for entangled neutral mesons, Nucl. Phys. B 744 (2006) 180 [hep-ph/0506025] [INSPIRE].

[35] L. Wolfenstein, Violation of time reversal invariance in K0 decays, Phys. Rev. Lett. 83 (1999) 911 [INSPIRE].

[36] A. Di Domenico (Ed.), Handbook on Neutral Kaon Interferometry at a $\phi$-factory, Frascati Phys. Ser. 43 (2007).

[37] J.R. Ellis, J.S. Hagelin, D.V. Nanopoulos and M. Srednicki, Search for Violations of Quantum Mechanics, Nucl. Phys. B 241 (1984) 381 [INSPIRE].

[38] J.R. Ellis, J.L. Lopez, N.E. Mavromatos and D.V. Nanopoulos, Precision tests of CPT symmetry and quantum mechanics in the neutral kaon system, Phys. Rev. D 53 (1996) 3846 [hep-ph/9505340] [INSPIRE].

[39] L.-F. Li and L. Wolfenstein, Current Algebra Analysis of CP Violations in $K \rightarrow 3 \pi$ Decay in the Six Quark Weinberg-Salam Model, Phys. Rev. D 21 (1980) 178 [INSPIRE].

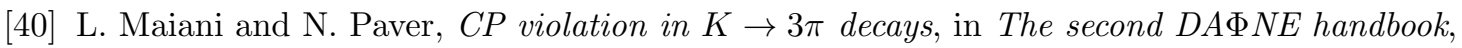
ed. L. Maiani, G. Pancheri, N. Paver, Vol.I p.51-62, INFN-LNF, Frascati (1995).

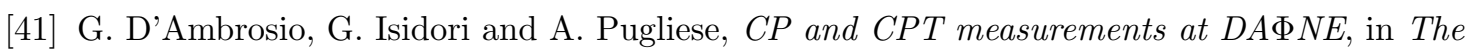
second DAФNE handbook, ed. L. Maiani, G. Pancheri, N. Paver, Vol.I p.63-95, INFN-LNF, Frascati (1995).

[42] KLOE collaboration, D. Babusci et al., A new limit on the CP-violating decay $K_{S} \rightarrow 3 \pi^{0}$ with the KLOE experiment, Phys. Lett. B 723 (2013) 54 [arXiv:1301.7623] [INSPIRE]. 